\title{
Correlation of very low and low frequency signal variations at mid-latitudes with magnetic activity and outer-zone particles
}

\author{
A. Rozhnoi ${ }^{1}$, M. Solovieva ${ }^{1}$, V. Fedun ${ }^{2}$, M. Hayakawa ${ }^{3}$, K. Schwingenschuh ${ }^{4}$, and B. Levin ${ }^{5}$ \\ ${ }^{1}$ Institute of Physics of the Earth, Russian Academy of Sciences, 10 B. Gruzinskaya, Moscow, 123995, Russia \\ ${ }^{2}$ Space Systems Laboratory, Department of Automatic Control and Systems Engineering, University of Sheffield, Sheffield, \\ S1 3JD, UK \\ ${ }^{3}$ University of Electro-Communications, Advanced Wireless Communications Research Center, 1-5-1 Chofugaoka, \\ Chofu Tokyo, 182-8585, Japan \\ ${ }^{4}$ Space Research Institute, Austrian Academy of Sciences, 6 Schmiedlstraße, 8042, Graz, Austria \\ ${ }^{5}$ Institute of marine geology and geophysics Far East Branch of Russian Academy of Sciences, 1B Nauki str., \\ Yuzhno-Sakhalinsk, 693022, Russia
}

Correspondence to: A. Rozhnoi (rozhnoi@ifz.ru)

Received: 31 May 2014 - Revised: 28 October 2014 - Accepted: 31 October 2014 - Published: 4 December 2014

\begin{abstract}
The disturbances of very low and low frequency signals in the lower mid-latitude ionosphere caused by magnetic storms, proton bursts and relativistic electron fluxes are investigated on the basis of VLF-LF measurements obtained in the Far East and European networks. We have found that magnetic storm $(-150<\mathrm{Dst}<-100 \mathrm{nT})$ influence is not strong on variations of VLF-LF signals. The anomalies with negative amplitude were registered during the main and recovery phases for several magnetic storms (mainly for three northernmost paths). The correlation between VLFLF signals and geomagnetic activity is rather weak even for these paths $(\approx 12-18 \%)$. Also, the correlation between magnetic activity and VLF signal variations recorded onboard the DEMETER satellite is not found. The significant influence of outer-zone particles (energetic particle sensor on board/Geostationary Operational Environmental Satellite (GOES) measurements) on the VLF-LF signal variations is found for almost half of the sub-ionospheric paths.
\end{abstract}

Keywords. Ionosphere (mid-latitude ionosphere)

\section{Introduction}

The measurements of sub-ionospheric very low and low frequency radio signals in the mid-latitude paths in the range of $15-50 \mathrm{kHz}$ are broadly used for the identification of earthquake electromagnetic precursors (see, e.g., Molchanov and
Hayakawa, 2008; Hayakawa et al., 2010; Hayakawa, 2011; Biagi et al., 2004, 2007; Rozhnoi et al., 2004, 2009). This band of electromagnetic waves is trapped between the lower ionosphere and the surface of the Earth, and reflected from the boundary between the upper atmosphere and lower ionosphere at altitudes of $\approx 70 \mathrm{~km}$ (daytime) and $\approx 90 \mathrm{~km}$ (nighttime). As a result, VLF-LF signals contain important information about the physical processes in the reflection region of the ionosphere and its variability. The selection and identification of the lower ionosphere perturbations, excited by seismic activity, tsunamis or volcanic eruptions from other possible external sources (e.g., planetary waves, cyclones and typhoons), are difficult problems. Additionally, to the Earth origin disturbances, the ionosphere could be forcing from above by magnetic storms, solar X-ray flashes, proton bursts and electron fluxes. The study of the amplitude and phase of VLF-LF anomalies of non-seismic origin is necessary for the reliable identification of seismic anomalies.

Previously, VLF phase and amplitude variations and their relationship with geomagnetic activity at mid-latitudes have been studied in the region $10.2-13.6 \mathrm{kHz}$ of the global navigation system "Omega" (see, e.g., Belrose and Thomas, 1968; Potemra and Rosenberg, 1973; Kikuchi, 1981; Sokolov, 2011). In general, the phase disturbances were registered during magnetic storms and the days following the recovery of the geomagnetic field. It was found that 


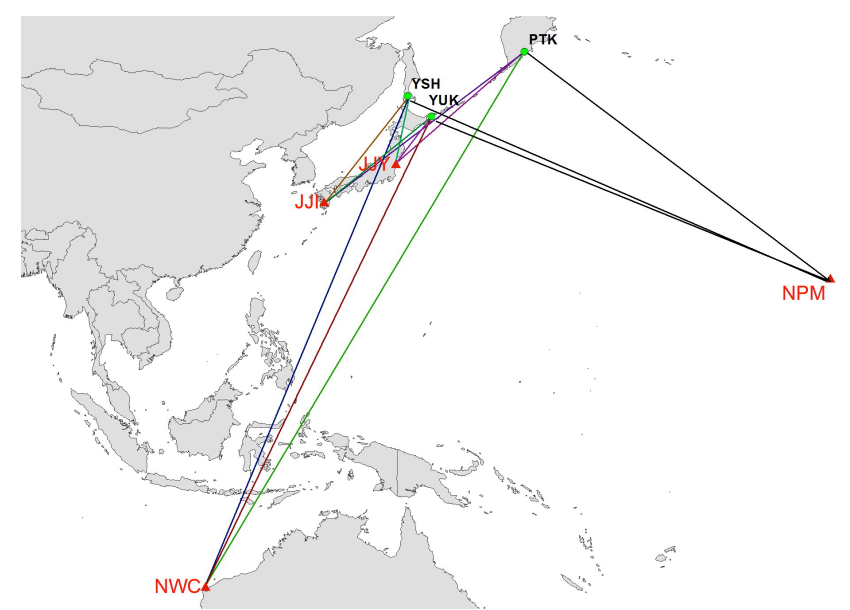

Figure 1. The map of the wave paths in the Far East network. The location of receivers in Petropavlovsk-Kamchatsky (PTK), YuzhnoSakhalinsk (YSH) and Yuzhno-Kurilsk (YUK) together with the position of the transmitters JJI $(22.2 \mathrm{kHz})$, JJY $(40 \mathrm{kHz})$, NWC $(19.8 \mathrm{kHz})$ and NPM $(21.4 \mathrm{kHz})$ are shown.

VLF anomalies can serve as an indirect indicator of energetic electron injection into the ionosphere.

System Omega was terminated in 1997 and therefore in our analysis, we used another navigation, as well as time service transmitters with different frequencies. The appearance of anomalies in the VLF-LF signal varies for different wave paths. They strongly depend on path length and its orientation relative to the magnetic field and signal frequency. For example, the analysis of the LF signal $(40 \mathrm{kHz})$ variations registered by the Petropavlovsk-Kamchatsky station has shown (see, e.g., Rozhnoi et al., 2006): (i) that LF signal anomalies are typical for the main phase of a magnetic storm in the night sector; (ii) the similarity of phase and amplitude anomalies of the LF signal to the structure of the simultaneously observed Pi3 geomagnetic pulsations; (iii) the moderate correlation between Dst index of magnetic activity and phase and amplitude anomalies of the LF signal; (iv) the strong correlation between proton bursts, fluxes of relativistic electrons registered on the board of the GOES satellite and characteristics of sub-ionospheric signal. Rozhnoi et al. (2006) have used another method, which was close to epoch analysis and gave several examples of case study analysis of the LF data during electron and proton fluxes. For further investigation of the VLF-LF variations and their relation to the space weather, the existing VLF-LF network was significantly extended in the Far East region and Europe. The new receivers were installed in Yuzhno-Sakhalinsk and YuzhnoKurilsk (Russia), Graz (Austria) and Sheffield (UK). This improvement and the following study are essential for (i) a better identification of electromagnetic precursors of earthquakes and (ii) for finding the lower ionosphere response to tsunamis and volcano eruptions. In this work, the case study and correlation analysis are applied for the investigation of

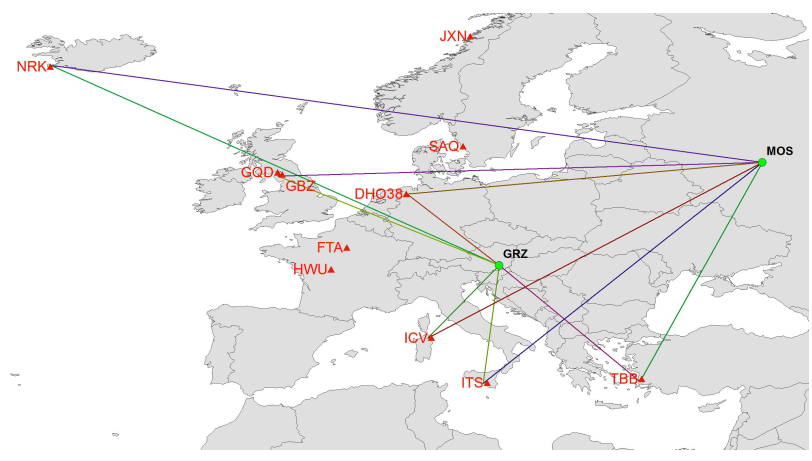

Figure 2. The same as in Fig. 1 but for the European network. The position of the receivers in Moscow (MOS) and Graz (GRZ) and different transmitters in Europe is shown on the map. Signals from six transmitters are analysed: NRK $(37.5 \mathrm{kHz})$, ICV $(20.27 \mathrm{kHz})$, ITS $(45.9 \mathrm{kHz})$, TBB $(26.7 \mathrm{kHz}), \mathrm{DHO}(23.4 \mathrm{kHz})$ and GBZ (19.58 kHz).

sub ionospheric VLF-LF signals propagation in various midlatitude paths for the Far East region and European networks. Besides ground-based observations, the correlation method is used to analyse the VLF variations recorded onboard the DEMETER satellite.

\section{Source data}

The analysed data were measured by the VLF-LF receivers settled in the Far East and European regions. Their locations are shown in Figs. 1 and 2, correspondingly. Receivers have been developed in New Zealand (http://ultramsk.com/) and they are able to provide continuous monitoring of the amplitude and phase of MSK (minimum shift key) modulated signals in a frequency range of $10-50 \mathrm{kHz}$ from several VLF-LF transmitters, simultaneously. Each receiver is designed to record signals with time resolutions in a range from $50 \mathrm{~ms}$ up to $60 \mathrm{~s}$. For the present study, we used $20 \mathrm{~s}$ sampling frequency measurements obtained from three Far East stations: Petropavlovsk-Kamchatsky (PTK) $\left(53.15^{\circ} \mathrm{N}\right.$; work since 2000), Yuzhno-Sakhalinsk (YSH) $\left(46.96^{\circ} \mathrm{N}\right.$; work since 2009) and Yuzhno-Kurilsk (YUK) $\left(44.035^{\circ} \mathrm{N}\right.$; work since 2011). The PTK station registers signals from four transmitters: JJI $\left(22.2 \mathrm{kHz}, 32.08^{\circ} \mathrm{N}\right.$, Japan), JJY $(40 \mathrm{kHz}$, $37.37^{\circ} \mathrm{N}$, Japan), NWC $\left(19.8 \mathrm{kHz}, 21.81^{\circ} \mathrm{S}\right.$, Australia) and NPM $\left(21.4 \mathrm{kHz}, 21.42^{\circ} \mathrm{N}\right.$, Hawaiian Islands). The YSH and YUK receivers are able to register signals from eight transmitters simultaneously. The wave paths from the JJI and JJY transmitters are at mid-latitudes; paths from the NPM transmitter are in mid- and low latitudes, and paths from the NWC transmitter are in low and mid-latitudes and cross the Equator. Note, these paths are long and cross the regions in which typhoons occur frequently.

To study the VLF-LF disturbances in the European region, we used data from Moscow (MOS) $\left(55.75^{\circ} \mathrm{N}\right.$; work 


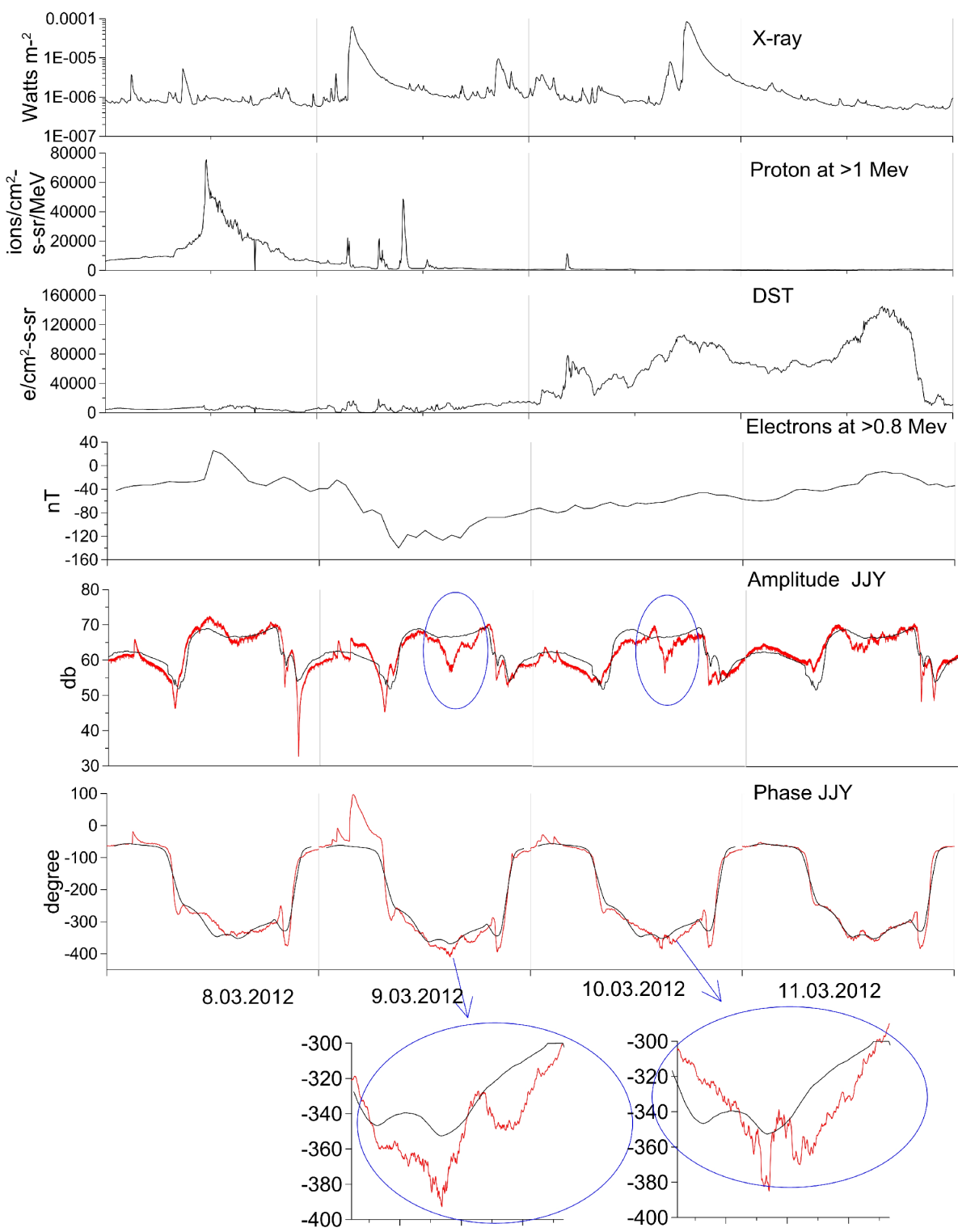

Figure 3. An example of the anomalies observed at the amplitude and phase of the JJY $(40 \mathrm{kHz})$ signal recorded in the PTK station during the magnetic storm on 9 March 2012. Three top panels show X-ray, outer-zone protons and electrons (GOES the satellite), and the next panel shows Dst index magnetic activity. In the two bottom panels, the amplitude and phase of the LF signal is shown. Red and black lines are the observed and monthly averaged signals, respectively. The circles highlight the anomalies in the amplitude and phase related to the magnetic storm.

since 2009) and Graz (GRZ) (47.065 N; work since 2009) stations. These stations can register signals from 12 transmitters deployed in Europe, Asia and America. For analysis, we selected the paths passing over seismo-active regions of southern Europe; i.e. the signals from TBB $(26.7 \mathrm{kHz}$, $37.4^{\circ} \mathrm{N}$ Turkey $)$, ICV $\left(20.27 \mathrm{kHz}, 40.92^{\circ} \mathrm{N}\right.$, Sardinia) and ITS $\left(45.9 \mathrm{kHz}, 37.11^{\circ} \mathrm{N}\right.$, Sicily) transmitters. As control (i.e. aseismic) paths, we have used signals from NRK $(37.5 \mathrm{kHz}$, $63.95^{\circ} \mathrm{N}$, Iceland $)$, DHO $\left(23.4 \mathrm{kHz}, 53.06^{\circ} \mathrm{N}\right.$, Germany $)$ and GBZ $\left(19.58 \mathrm{kHz}, 54.90^{\circ} \mathrm{N}, \mathrm{UK}\right)$ transmitters. All of these paths are located at mid-latitudes. In this research, we have used amplitudes of the VLF-LF signals, which do not require any additional preprocessing. Due to the complicity of VLFLF transmitter signal modulation, the phase identification requires special processing. For example, only in one case (see Fig. 3) has the phase preprocessing been used to evaluate phase anomalies. To find a correlation between space weather processes and VLF-LF signal variations, we have used Dst indexes, proton bursts and electron flux data from online available data sources (http://swdcwww.kugi.kyoto-u.ac.jp/ dstdir/index.html and http://spidr.ngdc.noaa.gov/spidr/). 


\section{Results}

\subsection{Correlation with magnetic activity}

The data from the YUK station are available only from August 2011, and therefore, for all other stations, we analysed data between August 2011 and September 2013. During this period, eleven strong magnetic storms occurred $(-150<$ Dst $<-100 \mathrm{nT})$, and corresponding detected anomalies in VLF-LF signals are summarised in Table 1.

The signal was considered as anomalous (disturbed) if its deviation from the monthly averaged value exceeded twice the standard deviation $(2 \sigma)$. For calculation of the monthly averaged value, we only used days with quiet signals. It is known that physical characteristics of the ionosphere are different at day and night. The daytime ionosphere is relatively stable, and its condition depends on solar activity. For example, the influence of X-rays emitted by solar energetic events on the phase of the signal - known as sudden phase anomalies (SPAs) - is shown in Fig. 3. Due to the high sensitivity of the ionosphere to the external disturbances caused by magnetic storms, particles fluxes, atmosphere circulation, seismic processes etc., the nighttime measurements are more informative. Since the time difference between the Far East and Europe is about $12 \mathrm{~h}$, the effects of magnetic storms for the European and Far Eastern regions were analysed separately. Some of them were considered in both regions if a sudden commencement (SC) and the main phase took place at different times or if the duration of the storm lasted for more than 1 day.

The magnetic storms with Dst $\approx-100 \mathrm{nT}$ neither influenced the VLF-LF signals on the European nor the Far East station networks (see Table 1). From an analysis of 24 wave paths, the anomalies in signals have only been found in five cases. In particular, the JJY signal (two events during the main and recovery phases), the NWC signal (two events during the main phase and one case during the recovery stage) and the JJI signal (one event during the main phase and two events during the recovery stage) were received at the Petropavlovsk-Kamchatsky station. These measurements confirm previous investigations by Kleimenova et al. (2004) and Rozhnoi et al. (2006). An example of such anomalies, observed simultaneously in the phase and amplitude of the JJY $(40 \mathrm{kHz})$ signal recorded at the PTK station during the magnetic storm on 9 March 2012 (Dst $\approx-140 \mathrm{nT}$ ), is shown in Fig. 3. The significant decreasing of the amplitude $(\approx 10 \mathrm{db})$ jointly with the phase variations of up to $40-50^{\circ}$, in relation to the averaged signal, were clearly seen during the night on 9-10 March 2012. An SPA in the LF signal induced by $\mathrm{X}$-rays was also visible in daytime measurements on 9 March 2012. Another X-ray event (10 March 2012) coincided with the evening terminator and therefore had no considerable effect on measured VLF-LF signals. An example of amplitude anomalies of the JJI $(22.2 \mathrm{kHz})$ signal during the magnetic storm on $8-9$ October 2012 (Dst $\approx-120$ nT)

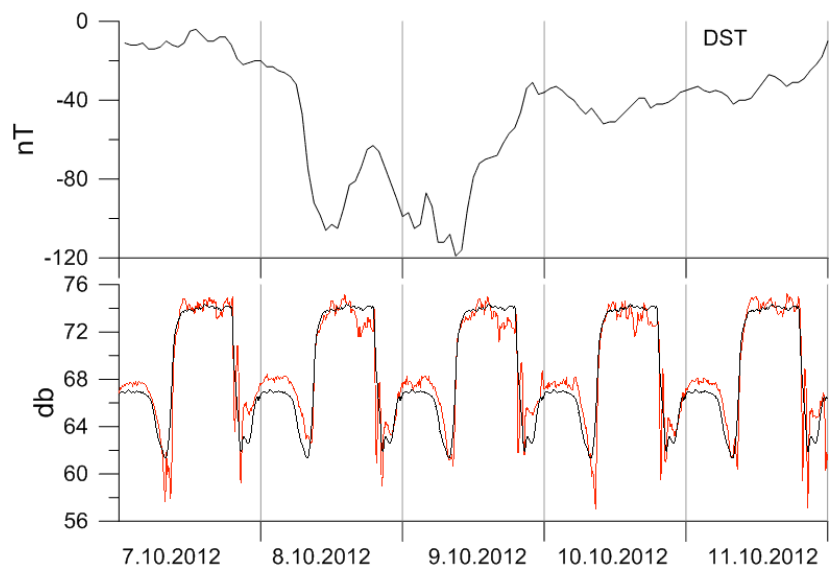

Figure 4. An example of the anomalies in the amplitude of the JJI $(22.2 \mathrm{kHz})$ signal during the magnetic storm on 8-9 October 2012. Red and black lines are the observed and monthly averaged signals, respectively.

is shown in Fig. 4. Unfortunately, this is not the MSKmodulated signal (whose phase cannot be recorded by receivers used in this study) and we have therefore only shown variations in amplitude. The negative anomalies at the amplitude of about $4 \mathrm{db}$ (i.e. more than $2 \sigma$ level for this very stable signal) are observed at nighttime on 8-10 March 2012.

In the European region (see Table 1), the anomalies during magnetic storms are found only for the NRK signal registered by Graz (six events) and the Moscow (three events) stations. Such a difference can be explained by difficulties of signal registration in the Moscow region due to the noisy electromagnetic environment of the megalopolis. Anomalies were also observed in the recovery phase; i.e. the "poststorm" effect.

We studied the sensitivity of VLF-LF signals to the geomagnetic activity by applying the correlation method. Two parameters have been used: (i) the residual signal of amplitude, calculated as the difference between nighttime real signal and monthly averaged signal (see, e.g., Rozhnoi et al., 2004), and (ii) the data series of Dst indexes during the year 2012. The daily variations between the maximum and minimum of Dst indexes, and the averaged residual amplitude of the VLF-LF signals in every wave path were used in computation. The cross-covariance function has been calculated at a time interval of \pm 10 days. We have found that the correlation of VLF-LF signals with Dst indexes is rather weak; i.e. $12-18 \%$ in three wave paths: JJY-PTK, NRK-GRZ and NRK-MOS. For example, the results for the PTK station are shown in Fig. 5. For the majority of wave paths, the correlations were not revealed. Therefore, the results of the ground-based data analysis show us the weak connection between VLF-LF measured signals and geomagnetic activity, and so, for further investigations, we included the 2004-2010 DEMETER satellite data (see, e.g., Parrot, 2002). This satellite had a near-polar circular orbit (inclination angle $86^{\circ}$; 
Table 1. Presence of anomalies in the VLF-LF signals during magnetic storms.

\begin{tabular}{lrlll}
\hline & & \multicolumn{3}{c}{ Paths with the anomalies in the signal } \\
\cline { 3 - 4 } Date, UT & Dst, nT & Main phase & Recovery phase & Night during the main phase \\
\hline 27 Sep 2011 & -103 & None & None & SC-the Far East-Europe \\
25 Oct 2011 & -135 & None & None & Europe \\
9 Mar 2012 & -140 & JJY-PTK, NWC-PTK & JJY-PTK, NWC-PTK & The Far East \\
24 Apr 2012 & -102 & NRK-MOS, NRK-GRZ & NRK-MOS, NRK-GRZ, JJY-PTK, JJI-PTK & Europe \\
16 Jul 2012 & -95 & None & None & The Far East \\
1 Oct 2012 & -144 & NRK-GRZ & None & Europe \\
8 Oct 2012 & -119 & JJY-PTK, NWC-PTK, JJI-PTK, NRK-GRZ & JJI-PTK, JJY-PTK, NRK-GRZ & 2 days \\
13 Oct 2012 & -100 & NRK-MOS, NRK-GRZ & NRK-MOS, NRK-GRZ & Europe \\
14 Nov 2012 & -108 & NRK-MOS, NRK-GRZ & NRK-GRZ & Europe \\
18 Mar 2013 & -105 & NRK-GRZ & NRK-GRZ & SC-the Far East, Europe \\
1 Jun 2013 & -120 & None & None & Europe \\
\hline
\end{tabular}

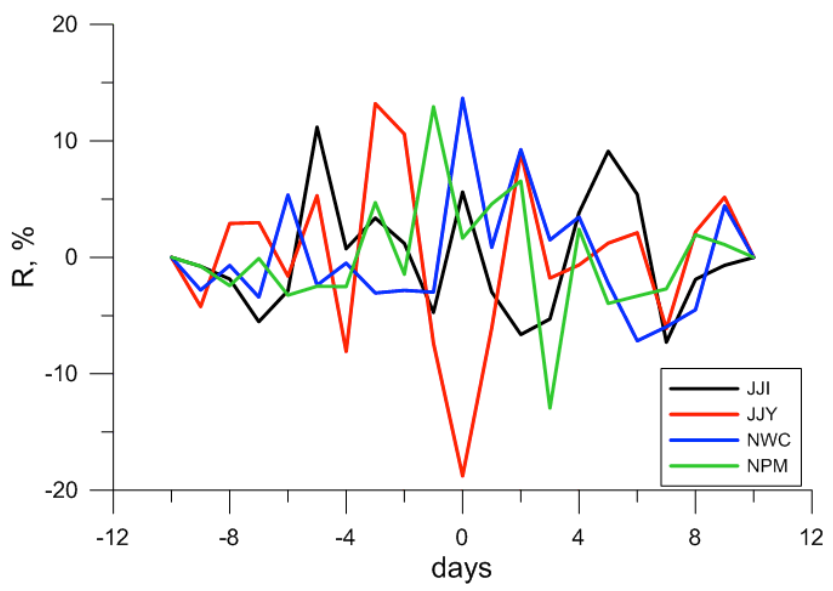

Figure 5. Cross-covariance functions for the average residual amplitude at nighttime of the signals received in PetropavlovskKamchatsky and MaxDst indexes calculated in the interval \pm 10 days. $Y$ axis is the correlation coefficient.

height $\approx 700 \mathrm{~km}$ ) and was located either shortly before the local noon (10:30 LT) or local midnight (22:30 LT) above the same point. DEMETER performed 14 orbits per day and was measured continuously between -65 and $+65^{\circ}$ of invariant latitude every $2 \mathrm{~s}$ in survey mode. For the analysis, we used data from the electric field experiment from the ICE instrument (Berthelier et al., 2006) in the lower frequency range $(20 \mathrm{~Hz}-20 \mathrm{kHz})$, recorded during the local night. Frequency resolution of the spectra in this range was about $19.5 \mathrm{~Hz}$. Since the DEMETER scientific mission had ended by December 2010, we chose a 1-year period of 2006 for analysis, which is comparable in magnetic activity with 2012.

Cross-covariance functions were calculated in two frequency ranges: (i) for low-frequency noise $150-1500 \mathrm{~Hz}$ and (ii) for the range of the NWC transmitter signal (19.6$20 \mathrm{kHz}$ ), which is most powerful in the VLF range. The spectrum broadening of the signal recorded by the satellite is about $\pm 200 \mathrm{~Hz}$ relative to the transmitter base frequency.
The reception zone of this signal covers most of the Eastern Hemisphere (Rozhnoi et al., 2007) and therefore signal analysis in the large area is possible. The whole reception signal area was divided into 12 zones with longitudinal width of every zone of $25^{\circ}$ that provided one orbit for every day. At latitude, the whole area was divided into three ranges: $-50^{\circ}<\Phi<-15^{\circ},-15^{\circ}<\Phi<15^{\circ}$ and $15^{\circ}<\Phi<50^{\circ}$ of magnetic latitudes. For further analysis, the satellite data were averaged in every zone identified above. Figures 6 and 7 show the cross-covariance functions between VLF data and Dst indexes calculated for 12 zones in frequency range 150 $1500 \mathrm{~Hz}$ and $19.6-20 \mathrm{kHz}$, respectively. The clear correlation of low-frequency noise $(150-1500 \mathrm{~Hz})$ with magnetic storm activity can be seen for all zones, but there was no significant correlation for the VLF transmitter signal.

\subsection{Correlation with proton bursts and relativistic electron fluxes}

To examine the sensitivity of VLF-LF signals in the midlatitude paths to the relativistic electron fluxes $(>2 \mathrm{MeV})$ and proton bursts $(0.6-2.4 \mathrm{MeV})$, the same correlation method was applied to the period of 2012. Data series of daily maximum characteristics recorded by energetic particle sensor on board/Geostationary Operational Environmental Satellite (GOES) were used for a cross-covariance functions calculation. A correlation with outer-zone particle fluxes was found for almost half of the paths in both the European and the Far East regions. The results for the MOS and GRZ stations are shown in Fig. 8. A correlation with particle fluxes is about $40 \%$ in the vicinity of the events. At the same time, the correlation analysis for 100 random data for every path (see Fig. 8 for path indication) does not reveal any apparent minimum (or maximum) in the vicinity of day "zero". Contradictory to the real data correlation, the random correlation does not vary for all intervals of analysis (i.e. \pm 10 days) and does not exceed -10 to $-12 \%$. Additionally, we examine how robust the correlations are. Pearson, bend and Spearman coefficients were found in range between -19 and $-25 \%$ 

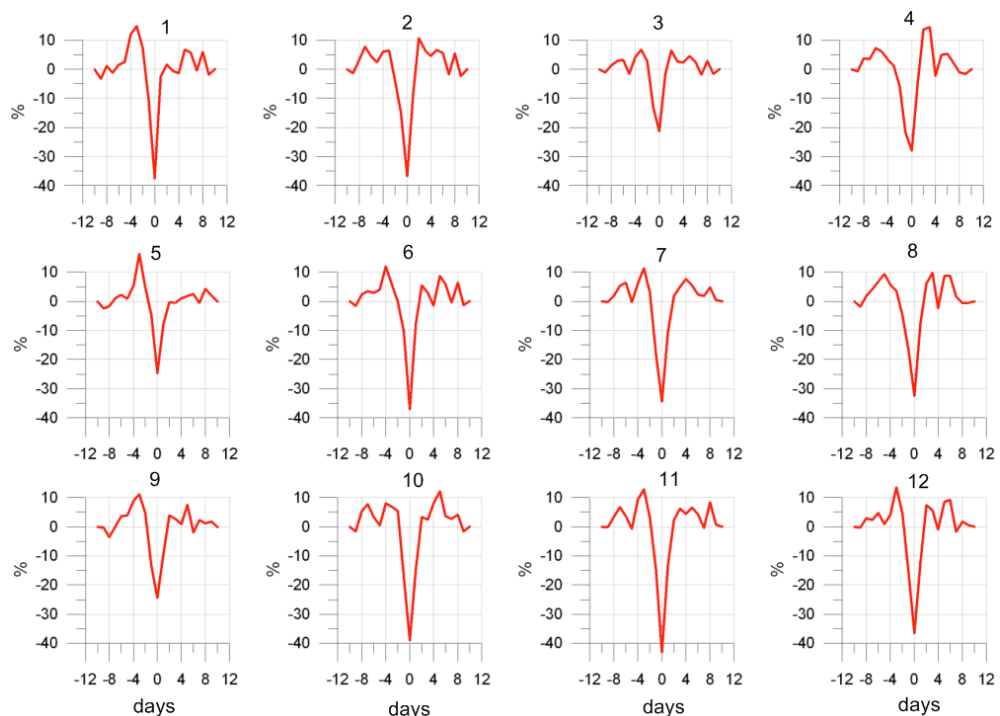

Figure 6. Cross-covariance function for the signal recorded onboard the DEMETER satellite in the frequency range 150-500 Hz averaged in the zones of analysis and Dst indexes. $Y$ axis is the correlation coefficient.
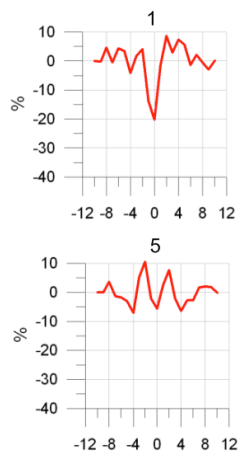

9

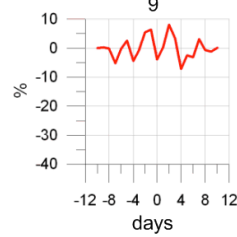

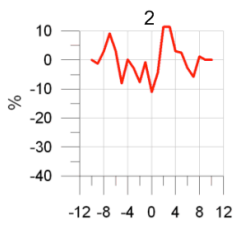
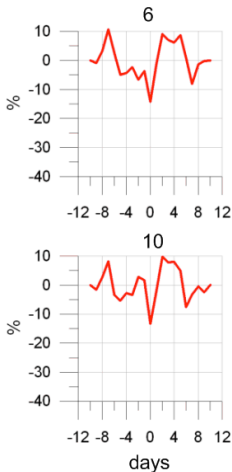
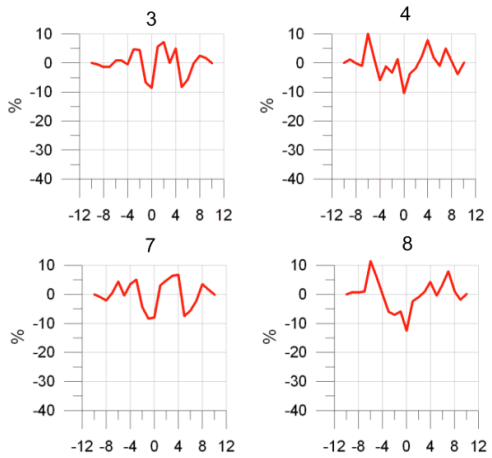

11

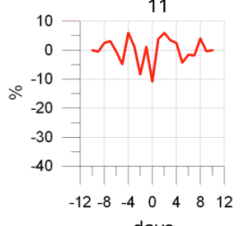

days
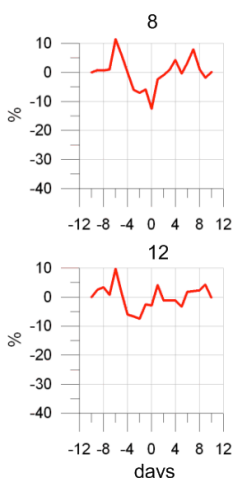

Figure 7. The same as in Fig. 6, but in the frequency range 19.6-20.0 kHz.

(depending on the path). For example, in NRK-MOS, paths were $-22,-21$ and $-25 \%$, correspondingly. It confirms that the correlation really exists.

Similar correlation (i.e. $\approx 40 \%$ ) was found between the low-frequency noise and geomagnetic activity in the DEMETER satellite data. We did not find any correlation dependence on the VLF-LF signals frequency or the relative position of transmitters and receivers. Our results indicate that the influence of particle fluxes on VLF-LF signals at midlatitudes is stronger than magnetic activity (Dst $>-150 \mathrm{nT}$ ) influence.

\section{Discussion and conclusions}

In this study, we investigated the response of the lower midlatitude ionosphere on magnetic activity and outer-zone particle fluxes (recorded by GOES satellite). The VLF-LF transmitter signals in a frequency range of $16-46 \mathrm{kHz}$ were analysed along 12 wave paths in the Far East and 12 paths in the European stations network. We have found that magnetic storms with $-150<$ Dst $<-100 \mathrm{nT}$ do not influence considerably on variations of the VLF-LF signals. The negative amplitude anomalies during the main and recovery phases have been revealed: (i) for three signals transmitted by JJY, JJI and NWC, and received by the PTK station; (ii) for one 

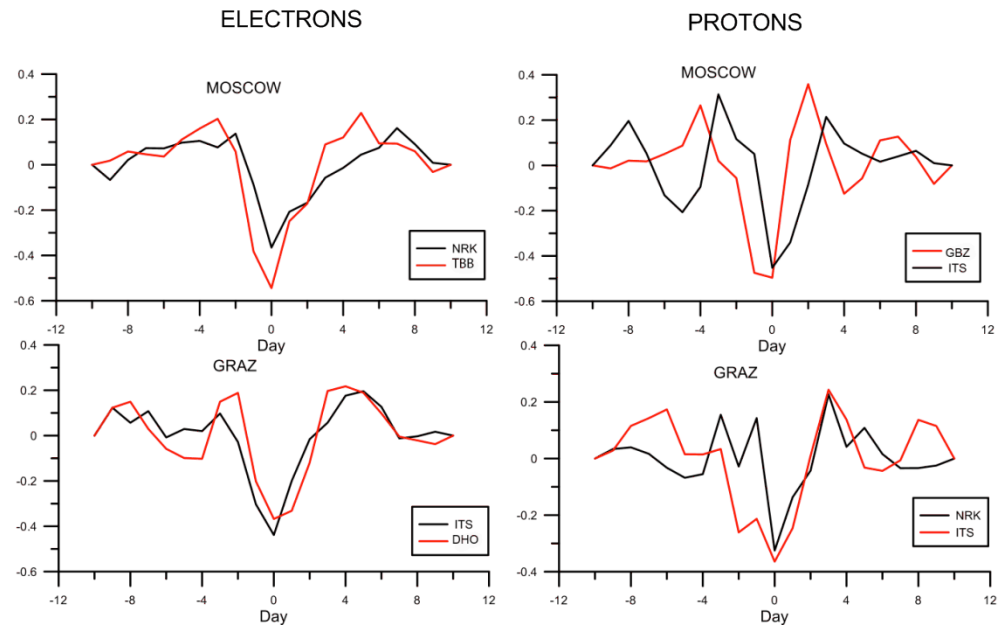

Figure 8. Cross-covariance functions for the average residual amplitude at nighttime of several signals receiving in the MOS and GRZ stations and the electron (left) and proton (right) fluxes (GOES). $Y$ axis is the correlation coefficient.

NRK signal received by MOS and GRZ stations in Europe. No correlation was found for any signals received in YSH or YUK stations. We have found that the correlation between VLF-LF signals and Dst indexes measured in 2012 is only about $12-18 \%$ for three wave paths (JJY-PTK, NRK-GRZ and NRK-MOS). For the majority of the wave paths, the correlation was not found. These results can be explained by taking into account the relative location of transmitters and receivers. As one can see from Fig. 1, the PTK is the northernmost station among all the Far East stations and, at the same time, the JJY transmitter is the northernmost transmitter in this region. Therefore, the JJY-PTK path is close to high latitude, where the influence of magnetic storm is strong. The European NRK transmitter is located near the polar circle that, undoubtedly, determines signal behaviour during magnetic variations.

The results of the ground-based measurements have been confirmed by an analysis of satellite DEMETER data. The analysis of electromagnetic signals in two frequency ranges has shown that geomagnetic activity is the main factor in the appearance of disturbances in low-frequency noise, but it does not influence the VLF transmitter signal.

The analysis of the influence of very strong magnetic storms (Dst $\approx-400 \mathrm{nT}$ ) on VLF-LF signal propagation was out of scope of this study due to the storms' non-occurrence during the observation period. The clear connection between the phase and amplitude variations of VLF-LF signals and strong magnetic perturbations has been investigated previously by, e.g., Schmitter (2010), Peter et al. (2006) and Rozhnoi et al. (2006). For example, very strong and long (about a week) anomalies in the amplitude and phase of the LF signal have been registered in the JJY-PTK and NRK-Bari (Italy) paths during a super strong magnetic storm (28-31 October 2003, Dst $<-400 \mathrm{nT}$ ) named "Halloween". The authors have identified the considerable increase of the night signal and simultaneous decrease of the day signal, resulting in the reduction of daily variations by half. In the southward Japanese wave paths, the anomalies were much weaker.

The influence of magnetic storms at mid-latitude paths was not revealed, but this factor cannot be ignored in using the VLF-LF signal method for the searching of electromagnetic precursors of earthquakes, as well as for the study of the lower ionosphere response to tsunamis and volcano eruptions. It should be noted that anomalies in VLFLF signals, detected during the recovery stage of magnetic storms and caused by seismic activity, were usually observed over several days. In particular, in the recovery phase, Dst index reaches its normal level, and therefore anomalies in VLF-LF signals in these periods can be mistakenly attributed to the seismic anomalies. For example, anomalies in two paths (i.e. JJY-PTK and JJI-PTK) were observed over 34 days (magnetic storm on 8 November 2012) and its recovery phase. Some earthquakes with a magnitude of $M=5.2$ 5.7 occurred in the sensitivity zones of these paths 3-5 days later. Without taking into account these natural factors, such measurements can be mistakenly interpreted as the influence of earthquakes.

Based on the analysis of phase measurements of the Omega VLF signal, the detailed investigation of the poststorm effect in mid-latitude paths has been reported by Sokolov (2011). The author has shown that anomalies in phase were observed in four paths up to 11-12 days during and after magnetic storms. Three different types of magnetic storms have been considered depending on the $B_{z}$ component of the interplanetary magnetic field (IMF) and solar wind velocity in the recovery phase. It was found that prolonged VLF phase anomalies at mid-latitudes are typical for an efficient type of magnetic storm ( $B_{z}$ component of the IMF at recovery phase is negative or close to zero, and solar wind velocity is more than or equal to $500 \mathrm{~km} \mathrm{~s}^{-1}$ ). 
The connection of VLF-LF signal disturbances with proton bursts and relativistic electron fluxes revealed that their influence on the signal propagation is stronger than the influence of geomagnetic activity. A correlation of about $40 \%$ was found for almost half of the considered paths. Therefore, we can conclude that the influence of outer-zone particles on the ionosphere D layer, and hence on VLF-LF signal propagation, is very important for the identification of earthquake precursors.

Acknowledgements. This research is partially supported the by Royal Society International Exchanges Scheme and RFBR under grant no. 13-05-92602 KOa.

Topical editor J. Wild thanks M. Kosch and A. J. Kavanagh for their help in evaluating this paper.

\section{References}

Biagi, P. F., Piccolo, R., Castellana, L., Maggipinto, T., Ermini, A., Martellucci, S., Bellecci, C., Perna, G., Capozzi, V., Molchanov, O. A., Hayakawa, M., and Ohta, K.: VLF-LF radio signals collected at Bari (South Italy): a preliminary analysis on signal anomalies associated with earthquakes, Nat. Hazards Earth Syst. Sci., 4, 685-689, doi:10.5194/nhess-4-685-2004, 2004.

Biagi, P. F., Castellana, L., Maggipinto, T., Maggipinto, G., Minafra, A., Ermini, A., Capozzi, V., Perna, G., Solovieva, M., Rozhnoi, A., Molchanov, O. A., and Hayakawa, M.: Decrease in the electric intensity of VLF/LF radio signals and possible connections, Nat. Hazards Earth Syst. Sci., 7, 423-430, doi:10.5194/nhess-7-423-2007, 2007.

Belrose, J. S. and Thomas, L.: Ionization in the middle latitude D-region associated with geomagnetic storms, J. Atmos. Terr. Phys., 30, 1397-1413, 1968.

Berthelier, J. J., Godefroy, M., Leblanc, F., Malingre, M., Menvielle, M., Lagoutte, D., Brochot, J. Y., Colin, F., Elie, F., Legendre, C., Zamora, P., Benoist, D., Chapuis, Y., and Artru, J.: ICE, The electric field experiment on DEMETER, Planet. Space Sci., 54, 456-471, 2006.

Hayakawa, M.: Probing the lower ionospheric perturbations associated with earthquakes by means of subionospheric VLF/LF propagation, Earthquake Sci., 24, 609-637, 2011.

Hayakawa, M., Kasahara, Y., Nakamura, T., Muto, F., Horie, T., Maekawa, S., Hobara, Y., Rozhnoi, A. A., Solivieva, M., and Molchanov, O. A.: A statistical study on the correlation between lower ionospheric perturbations as seen by subionospheric VLF/LF propagation and earthquakes, J. Geophys. Res., 115, A09305, doi:10.1029/2009JA015143, 2010.
Kikuchi, T.: VLF phase anomalies associated with substorm, Mem. Nat. Inst. Polar. Res., Spec. Issue, 18, 3-23, 1981.

Kleimenova, N. G., Kozyreva, O. V., Rozhnoi, A. A., and Solovieva, M. S.: Variation in the VLF signal parameters on the AustraliaKamchatka radio path during magnetic storms, Geomagn. Aeronomy+, 44, 354-361, 2004.

Molchanov, O. A. and Hayakawa, M.: Seismo Electromagnetics and Related Phenomena: History and Latest results, TERRAPUB, Tokyo, 189 pp., 2008.

Parrot, M.: The micro-satellite DEMETER: data registration and data processing, Seismo-Electromagnetics (LithosphereAtmosphere-Ionosphere Coupling), edited by: Hayakawa, M. and Molchanov, O., TERRAPUB, Tokyo, 660-670, 2002.

Peter, W. B., Chevalier, M. W., and Inan, U. S.: Perturbations of midlatitude subionospheric VLF signals associated with lower ionospheric disturbances during major geomagnetic storms, J. Geophys. Res., 111, A03301, doi:10.1029/2005JA011346, 2006.

Potemra, T. A. and Rosenberg, T. J.: VLF propagation disturbances and electron precipitation at mid-latitudes, J. Geophys. Res., 78, 1572-1580, 1973.

Rozhnoi, A., Solovieva, M. S., Molchanov, O. A., and Hayakawa, M.: Middle latitude LF $(40 \mathrm{kHz})$ phase variations associated with earthquakes for quiet and disturbed geomagnetic conditions, Phys. Chem. Earth, 29, 589-598, 2004.

Rozhnoi, A. A., Solovieva, M. S., Molchanov, O. A., Hayakawa, M., Maekawa, S., and Biagi, P. F.: Sensitivity of LF signal to global ionosphere and atmosphere perturbations in the network of stations, Phys. Chem. Earth, 31, 409-415, 2006.

Rozhnoi, A., Molchanov, O., Solovieva, M., Gladyshev, V., Akentieva, O., Berthelier, J. J., Parrot, M., Lefeuvre, F., Hayakawa, M., Castellana, L., and Biagi, P. F.: Possible seismo-ionosphere perturbations revealed by VLF signals collected on ground and on a satellite, Nat. Hazards Earth Syst. Sci., 7, 617-624, doi:10.5194/nhess-7-617-2007, 2007.

Rozhnoi, A., Solovieva, M., Molchanov, O., Schwingenschuh, K., Boudjada, M., Biagi, P. F., Maggipinto, T., Castellana, L., Ermini, A., and Hayakawa, M.: Anomalies in VLF radio signals prior the Abruzzo earthquake $(M=6.3)$ on 6 April 2009, Nat. Hazards Earth Syst. Sci., 9, 1727-1732, doi:10.5194/nhess-91727-2009, 2009.

Schmitter, E. D.: Remote auroral activity detection and modeling using low frequency transmitter signal reception at a midlatitude site, Ann. Geophys., 28, 1807-1811, doi:10.5194/angeo-281807-2010, 2010.

Sokolov, S. N.: Magnetic storms and their effects in the lower ionosphere: differences in storms of various types, Geomagn. Aeronomy+, 51, 741-752, 2011. 